\title{
Numerical Control Turning Principle and Realization Method of Cam Shape Surface
}

\author{
Dongfang Hu and Jianwei Guo \\ School of Mechatronics Engineering, Henan University of Science and Technology, Luoyang \\ 471003, China \\ ahdf@haust.edu.cn, bguojianwei1991@yeah.net
}

\begin{abstract}
Keywords: Cam shaft; Turning principles; Noncircular turning; Shape surface; Grinding
\end{abstract}
\begin{abstract}
In order to illustrate the numerical control turning principle of camshaft, analyzed the influence on the work-piece quality and the tool life by cutting speed and tool working angle in noncircular turning process. Established the mathematical model of the relationship between cutting speed and tool working angle. Then introduced the design concept of constant line speed turning. Quantitatively analyzed the regular pattern of the tool working angle with the variation of circumferential position in noncircular section of part. In order to ensure the quality of the camshaft surface, proposed the realization scheme of the swing of the cam profile in the non-circular turning reciprocating motion, and the mathematical model of the constant speed linear grinding camshaft is deduced, as the ultimate guarantee of cam profile quality grinding theory basis.
\end{abstract}

\section{Introduction}

Due to the presence of irregularly rotating surfaces of the camshaft, the increase in turning speed has been limited ${ }^{[1]}$. With only a "soft" profiling (numerical control program) control, it is difficult to solve fundamentally the problem of slow speed, while it could not use the limit lathe processing hard modeling of flexible control. So it need find another way to improve the process ${ }^{[2]}$. Proper equipment adjustment and optimization tool angle and other auxiliary equipment to improve the cutting speed. Wang Xiankui, beginning in 2003, tsinghua university, who carried out the numerical control turning system key technology of the camshaft research, radial motion of the cutting tool and cutting tool angle for real-time control, the cam shaft turning and cylindrical turning can be done at the same time ${ }^{[3]}$.

Cutting angle and cutting speed are different with the location of cam outline processing, it need to take measures to make sure cutting angle basically constant in the process. Zheng Mengkun of huazhong university of science and technology, and others carried out optimization design in high precision camshaft grinding machine of grinding wheel rack, put forward kinematics model of constant camshaft grinding, it has important significance to ensure the quality of cam profile in $2012^{[4]}$. Although the camshaft turning has a larger development, but based on the processing means of economy, it still need to use grinding camshaft as finishing the final process ${ }^{[5]}$.

\section{The Cam Turning Molding Technology}

In order to achieve this movement, it can be used by mould processing, it can also use the method of numerical control, so-called electronic modeling or soft modeling ${ }^{[6]}$.

Cutting Tool High Frequency Repetitive Motion. In order to achieve the reciprocating movement of cutting tool, choosing dc motor driven tool to make radial reciprocating movement. When the tool processing like figure 1. Motor drive the cutter along the $\mathrm{OO}$ 'direction of reciprocating motion, the trajectory of cutting tool moving can use cam radius vector and roller radius in OO' direction of horizontal projection minus the roller radius and the CAM base circle radius to represented.

$$
X(t)=O O^{\prime}-\mathrm{r}_{0}-\mathrm{r}_{1}
$$

Since $O O^{\prime}=r_{t} \cos \alpha-r_{1} \cos \theta$, therefore 


$$
X(t)=r_{t} \cos \alpha-r_{1} \cos \theta-r_{0}-r_{1}
$$

in the equation, $r_{t}$ is cam radiusvector, $r_{1}$ is follower radius, according to the equation (1), when $t=0$, $\mathrm{r}_{\mathrm{t}}=\mathrm{r}_{0}$.

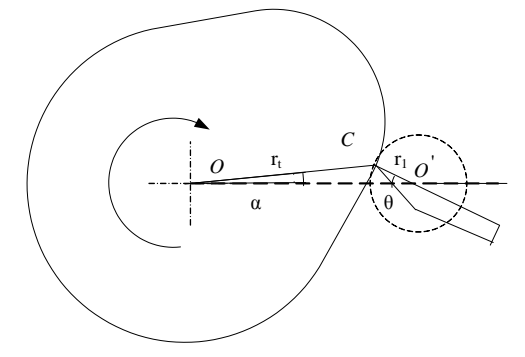

Figure.1The cam turning state diagram

By the trajectory in figure 1, according to the law of cosines OO 'center distance the relationship between angle of cam with alpha $\alpha$ can be deduced:

$O O^{\prime 2}-2 O O^{\prime} \times O C \times \cos \alpha+O C^{2}=O^{\prime} C^{2}$

In the cam, radius vector $\mathbf{r}_{\mathbf{t}}$ has function relationship with $\omega \mathbf{t}$, it is defined by the trajectory of cam contour, assuming:

$O C=r_{t}=g(\omega t), O^{\prime} C=r_{1}$

The relationship between angle $\alpha$ with spindle speed are also depends on the cam contour, assuming: $\alpha=\phi(2 \pi \omega t)$

The expression of $\mathrm{X}(\mathrm{t})$ can be acquired by (1), (3), (4) (take the cam base circle with a roller follower and tangent of center distance is zero):

$$
x(t)=g(\omega t) \times \cos [\phi(2 \pi \omega t)]-\sqrt{\mathrm{r}^{2}{ }_{1}-g^{2}(\omega t) \times \sin ^{2}[\phi(2 \pi \omega t)]}-\mathrm{r}_{1}-\mathrm{r}_{0}
$$

When machining cam turning tool, therefore, the trajectory of high frequency reciprocating motion driven by the linear motor is $\mathrm{x}(\mathrm{t})$.

Tool Oscillation Real-Time Control. In cutting tool machining process, the cutting tool be adapt to the cam contour real-time swing, so it can ensure the former and behind cutting tool angle are basic constant ${ }^{[7]}$, this is aspect which needs to be addressed in the process. By the cutting diagram shown in figure 2, the cutting tool with roller center $\mathrm{O}$ 'real-time swinging for the rotary centers and $\mathrm{O}^{\prime} \mathrm{C}$ perpendicular to the cross section of the cam contour. When turning the cam, turning speed direction should be the cutting point on the cam contour tangent direction, such as cutting plane is seen $\mathrm{t}-\mathrm{t}$ plane, and synthesis of the velocity vector is on the plane $t-t$; The work base and synthetic velocity direction is vertical each other, $\mathrm{n}-\mathrm{n}$ plane is the base position, it is also normal direction in turning point, the sharp of cutter point positive pressure direction is inside the base. By the mechanical principles on the expression of pressure angle of cam, the pressure angle of cam mechanism is positive pressure direction by the push rod and the angle between the push rod tip speed direction, the tool swinging angle $\theta$ and cutting base and tool linear motion direction angle is equal, so $\theta$ is the cutting point of pressure angle currently, find the relationship between the pressure angle and the spindle rotation angle, tool oscillation real-time trajectory can be gotten. $\mathrm{D}$ in combination cam related knowledge, the graphic as a cam for roller slewing center $\mathrm{O}$ 'speed instantaneous center ${ }^{[8]}$. Turning tool around $\mathrm{O}$ 'rotation, at this time: 


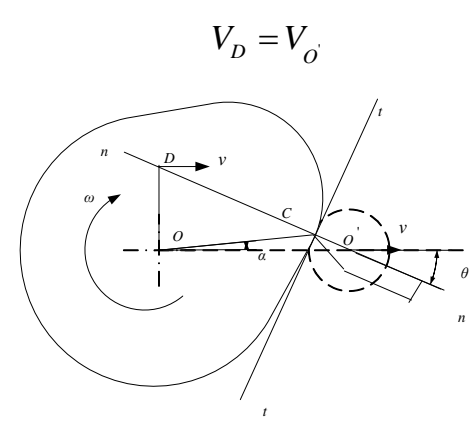

Figure. 2 geometric relationships between turning tool with the cam when tool swinging

At this time by graphic geometric relationships available:

$$
\begin{aligned}
& \tan \theta=\frac{O D}{O O^{\prime}}=\frac{v}{\omega O O^{\prime}} \\
& O O^{\prime}=O C \times \cos \alpha-\sqrt{O^{\prime} C^{2}-O C^{2} \times \sin ^{2} \alpha} \\
& =r(t) \times \cos \alpha-\sqrt{O^{\prime} C^{2}-O C^{2} \times \sin ^{2} \alpha} \\
& =g(\omega t) \cos \alpha-\sqrt{r^{2}{ }_{1}-g^{2}(\omega t) \times \sin ^{2}[\phi(2 \pi \omega t)]}
\end{aligned}
$$

And there was a: $v=\frac{d x(t)}{d(t)}$

Therefore

$$
\tan \theta=\frac{O D}{O O^{\prime}}=\frac{v}{\omega O O^{\prime}}
$$

$$
=\frac{d x(t)}{d(t)} \times \frac{1}{\omega} \times \frac{1}{\left.g(\omega t) \cos \alpha-\sqrt{r^{2}{ }_{1}-g^{2}(\omega t) \times \sin ^{2}[\phi(2 \pi \omega t)}\right]}
$$

In the equation, $x(t)=g(\omega t) \times \cos [\phi(2 \pi \omega t)]-\sqrt{\mathrm{r}{ }_{1}-g^{2}(\omega t) \times \sin ^{2}[\phi(2 \pi \omega t)]}-\mathrm{r}_{1}-\mathrm{r}_{0}$

To ensure that angle cam, it must according to the type (3-6) for real-time control tool swinging angle in turning process. In the back of the practice, adopted the method of double linear motor driven, by moving around the two relative speed is different, to realize the adapt to change of cam contour line for cutter deflection angle.

\section{The Camshaft Grinding Mathematical Model of Constant Line Speed}

From the processing means of economy, grinding is needed to ensure the quality of cam profile finally [9].

Camshaft grinding processing means the constant linear velocity of grinding and grinding force in grinding process is relatively stable grinding. When processing cam, by the grinding wheel rack $\mathrm{C}$ axis of $\mathrm{X}$ to mobile and spindle rotating two axes linkage to the common form cam contour surface, principle of two axes linkage motion is determined by the constant line speed mathematical model ${ }^{[10]}$. 


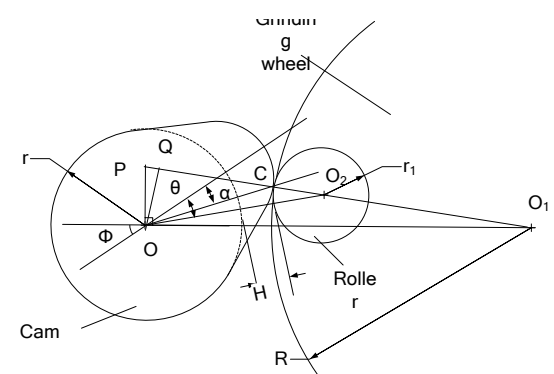

Figure 3 cam grinding schematic diagram

As shown in figure 3 is based on the roller follower cam grinding schematic diagram of the model, which is $\mathrm{O}$ is cam base circle's center, $\mathrm{O} 1$ is center for grinding wheel, $\mathrm{O} 2$ is tappet roller center, $\mathrm{r}$ is the radius of base circle, $\mathrm{r} 1$ is tappet roller radius, $\mathrm{R}$ is the radius of the grinding wheel, $\mathrm{H}$ is the lift roller. $\Phi$ is cam rotation angle, $\theta$ is the angle lifter, $\alpha$ is grinding points around the corner

As can be seen from Figure 3-17: $\overline{O O_{2}}=\mathrm{r}+H+\mathrm{r}_{1}$

Assuming that the lift equation is $\mathrm{H}(\theta)$, make $\overline{O P} \perp \overline{O O_{1}}$, therefore:

$$
\overline{O P}=-\frac{d H(\theta)}{d(\theta)}
$$

Thus: $\quad \angle O O_{1} P=\arctan \left(\frac{\overline{O P}}{\overline{O O_{1}}}\right)$, make $\overline{O Q} \perp O_{2} O_{1}$, therefore:

$$
\begin{aligned}
& \overline{O Q}=\overline{O O_{1}} \sin \angle O O_{1} P \\
& \overline{O_{1} Q}=\overline{O O_{1}} \cos \angle O O_{1} P \\
& \overline{Q C}=\overline{O_{1} Q}-\mathrm{R} \\
& \overline{Q O_{2}}=\overline{Q C}+\mathrm{r}_{1}
\end{aligned}
$$

It can be obtained from the above: $\overline{O C}=\sqrt{\overline{O Q^{2}}+\overline{Q C^{2}}}$

Assuming the $S_{(X)}$ is displacement of the wheel frame, $\Phi$ is the corresponding cam angle

$$
\begin{aligned}
& S_{(X)}=\sqrt{\overline{O Q^{2}}+\overline{Q O_{1}^{2}}}-R-\mathrm{r} \\
& \phi=\theta+\angle O_{1} O O_{2}=\theta+\left(\angle O O_{2} P-\angle O O_{1} P\right)=\theta+\arctan \left(\frac{\overline{O Q}}{Q O_{2}}\right)-\arctan \left(\frac{\overline{O P}}{O O_{1}}\right) \\
& \alpha=\theta-O_{2} O C=\theta-\left(\angle Q C O-\angle O O_{2} P\right)=\theta-\arctan \left(\frac{\overline{O Q}}{\overline{Q C}}\right)+\arctan \left(\frac{\overline{O P}}{O O_{1}}\right)
\end{aligned}
$$

(8)

If the cam's shape function is $S_{(\alpha)}$, the angular velocity of the cam is $\omega$, then the displacement line velocity of the grinding point is:

Among them

$$
\begin{aligned}
& V_{C}=\frac{d s(\alpha)}{d(t)}=\frac{d s}{d \alpha} \times \frac{d \alpha}{d \phi} \times \frac{d \phi}{d t} \\
& \frac{\mathrm{ds}(\alpha)}{\mathrm{d} \alpha}=\sqrt{\overline{O C}^{2}+\left(\frac{\partial \overline{O C}}{\partial \alpha}\right)^{2}}, \frac{d \phi}{d t}=\omega .
\end{aligned}
$$

Assuming you set a certain corner of the round speed is ${ }^{\omega_{0}}$, if make the grinding point of the line speed same as the base, so: 
$V_{C}=\omega_{0} \times r$

Which is : $\quad \frac{d s}{d \alpha} \times \frac{d \alpha}{d \phi} \times \omega_{c}=\omega_{0} \times r$

It is possible to obtain the angular velocity $\omega_{c}$ in any one grinding points $\mathrm{C}$ at the time of cam machining, because the rotating speed $\omega=\frac{2 \pi n}{60}$, thus $\mathrm{C}$ rotating speed is

$$
N_{\mathrm{c}}=\frac{\omega_{0} \times \mathrm{r}}{2 \pi \sqrt{\overline{O C}^{2}+\left(\frac{\partial \overline{O C}}{\partial \alpha}\right)^{2}} \frac{\mathrm{d} \alpha}{\mathrm{d} \phi}} \times 60
$$

It can be seen from the above formula when the cam and grinding wheel are given, the cam speed $\omega 0$ is know, you can find the grinding wheel $\mathrm{C}$ on the cutting point speed NC. In other words, the rotational speed of the cutting point $C$ on the grinding wheel is determined by the radius of curvature of the cam and the grinding wheel and the cam angular velocity.

\section{Summary}

This chapter focuses on the principle of numerical control turning of camshaft, and established the numerical control turning model. Numerical control turning cam is to achieve two interrelated movement, one is the radial reciprocating motion in the radial direction, and the other is to ensure the stability of the turning, and require the turning tool to adapt to the cam profile reciprocating swing. In addition, in order to ensure the stability of the quality of the cam profile and get a good working condition of the turning tool .Introduced a design concept of constant line speed. Find the function relationship between the tool deflection angle and the spindle speed. On the basis of establishing the numerical control turning model, further established the mathematical model of the constant linear speed grinding, which laid the foundation for the ultimate guarantee of the quality of the cam profile.

\section{Acknowledgements}

The authors gratefully acknowledge the National Nature Science Foundation (No. 51405135).

\section{References}

[1] Zhao Qinde. Numerical turning method for camshaft[J]. Numerical technology and application, 2012, (6): 1-2.

[2] Chang Xinge, Liu Hongli. Camshaft turning craft method[J]. modern part, 2012, (9): 56-57.

[3] Wu Dan, Wang Xiankui, Zhao Tong. Non-circular turning cutter movement realization methods[J]. Tsinghua University(natural science edition) 2003, 43(11): 1472-1475.

[4] Zheng Mengkun. The optimize of high-speed and high accuracy camshaft grinding wheel frame[D]. Wu Han. Huazhong university of science and technology. 2012.

[5] Gu Feng. Cam process craft based on turning and milling technology[D]. Shen Yang. Northeastern University. 2011.

[6] Zhao Deqin. Numerical control turning method of camshaft[J]. numerical technology application and development, 2012, (6): 1-2.

[7] Hong Meiqin. Realize cutter angle in real-time control swing tools frame design in numerical control turning cam[J]. mechanical engineer, 2014, (10): 137-139.

[8] Sun Heng, Chen Zuomo. Mechanical theory[M]. higher education press, 1996.

[9] Xi Aijun. Cam surface roughness finishing technology research[J]. modern auto power, 2012 (2): 53-56.

[10] Sun Mei. Research on camshaft constant line speed process mathematic model optimize[J]. GuangXi light industry2011, 27(2): 52-53. 